\title{
SET PARTITIONS WITHOUT BLOCKS OF CERTAIN SIZES
}

\author{
JOSHUA CULVER AND ANDREAS WEINGARTNER
}

\begin{abstract}
We give an asymptotic estimate for the number of partitions of a set of $n$ elements, whose block sizes avoid a given set $\mathcal{S}$ of natural numbers. As an application, we derive an estimate for the number of partitions of a set with $n$ elements, which have the property that its blocks can be combined to form subsets of any size between 1 and $n$.
\end{abstract}

\section{INTRODUCTION}

Let $B_{n}$ be the $n$-th Bell number, that is the number of set-partitions of a set with $n$ distinct elements. For example, $B_{3}=5$ because there are five set-partitions of $\{a, b, c\}$ :

$$
\{\{a, b, c\}\},\{\{a\},\{b, c\}\},\{\{b\},\{a, c\}\},\{\{c\},\{a, b\}\},\{\{a\},\{b\},\{c\}\} .
$$

We say that the partition $\{\{a\},\{b, c\}\}$ has the block $\{a\}$ of size 1 and the block $\{b, c\}$ of size 2 . Blocks of size zero (empty blocks) are not allowed.

Let $\mathcal{S} \subset \mathbb{N}$ and let $B_{n, \mathcal{S}}$ denote the number of partitions of a set with $n$ elements, whose block sizes are not in $\mathcal{S}$. If $\mathcal{S}=\{1,2, \ldots, m\}$, we write $B_{n, m}=B_{n, \mathcal{S}}$, the number of partitions of a set with $n$ elements, all of whose block sizes are greater than $m$. We shall call such partitions m-rough. For example, $B_{3,1}=B_{3,2}=1$. We clearly have $B_{n, 0}=B_{n}$. We define $B_{0, \mathcal{S}}=1$ for all $\mathcal{S}$.

Throughout this paper, we write $r=r(n)$ to denote the solution of

$$
r e^{r}=n .
$$

The function $r$ is called Lambert- $W$ function or product logarithm, and it can be approximated with the asymptotic formula (see [1])

$$
r=\log n-\log \log n+\frac{\log \log n}{\log n}+O\left(\left(\frac{\log \log n}{\log n}\right)^{2}\right) .
$$

Let

$$
\alpha(z)=\alpha_{\mathcal{S}}(z)=\sum_{k \in \mathcal{S}} \frac{z^{k}}{k !} .
$$

Our first result is an asymptotic estimate for $B_{n, \mathcal{S}}$, derived from Cauchy's residue theorem and the saddle point method.

2010 Mathematics Subject Classification. Primary 05A18; Secondary 05A16. 
Theorem 1. Let $\eta_{1}=0.1866823 \ldots, \eta_{2}=2.1555352 \ldots$ be the two real solutions of $\eta(1-\log \eta)=1 / 2$ and let $0<\delta_{1}<\eta_{1}<\eta_{2}<\delta_{2}$. We have

$$
B_{n, \mathcal{S}}=\frac{n ! \exp \left(e^{r}-1-\alpha(r)\right)}{r^{n} \sqrt{2 \pi r(r+1) e^{r}}}\left(1+O\left(\frac{1+\left(\alpha^{\prime}(r)\right)^{2}}{e^{r}}\right)\right),
$$

uniformly for sets $\mathcal{S}$ with $\mathcal{S} \cap\left[\delta_{1} r, \delta_{2} r\right]=\emptyset$.

Lemma 3 shows that the relative error term in Theorem 1 approaches zero as $n \rightarrow \infty$, uniformly for sets $\mathcal{S}$ with $\mathcal{S} \cap\left[\delta_{1} r, \delta_{2} r\right]=\emptyset$. The constant factor implied in the big-O notation depends only on the choice of the constants $\delta_{1}, \delta_{2}$, but does not depend on $n$ or $\mathcal{S}$. On the other hand, if $\mathcal{S} \cap\left[\gamma_{1} r, \gamma_{2} r\right] \neq \emptyset$, for constants $\eta_{1}<\gamma_{1}<\gamma_{2}<\eta_{2}$, then the error term in Theorem 1 grows unbounded as $n \rightarrow \infty$. In this case, it seems that a different method is needed to determine the asymptotic behavior of $B_{n, \mathcal{S}}$.

The contribution to $\alpha(r)$ from $k \geq(e+\varepsilon) r$ is $o(1)$ as $n \rightarrow \infty$, if $\varepsilon>0$. Thus, $B_{n, \mathcal{S}} \sim B_{n, \mathcal{S}^{\prime}}$ if $\mathcal{S}$ and $\mathcal{S}^{\prime}$ avoid $\left[\delta_{1} r, \delta_{2} r\right]$ and differ only on $((e+\varepsilon) r, \infty)$.

If $\max \mathcal{S} \leq r$, the occurrence of $\alpha^{\prime}(r)$ in the error term of Theorem 1 can be estimated as in equation (3), with $i=1$, to obtain $\alpha^{\prime}(r)<(r / m)^{m-1} e^{m}$, where $m=\max \mathcal{S}$.

Corollary 1. With $\delta_{1}$ as in Theorem 1 and $m=\max \mathcal{S}$, we have

$$
B_{n, \mathcal{S}}=\frac{n ! \exp \left(e^{r}-1-\alpha(r)\right)}{r^{n} \sqrt{2 \pi r(r+1) e^{r}}}\left(1+O\left(\frac{(e r / m)^{2 m-2}}{e^{r}}\right)\right),
$$

uniformly for sets $\mathcal{S}$ with $1 \leq m \leq \delta_{1} r$.

If $\mathcal{S}=\emptyset$, then $\alpha(r)=0$ and Theorem 1 simplifies to the known asymptotic estimate for the Bell numbers $B_{n}$ (see Moser and Wyman [6]):

Corollary 2. We have

$$
B_{n}=\frac{n ! \exp \left(e^{r}-1\right)}{r^{n} \sqrt{2 \pi r(r+1) e^{r}}}\left(1+O\left(e^{-r}\right)\right) .
$$

Dividing the estimate in Theorem 1 by the one in Corollary 2 leads to the following result.

Corollary 3. Let $\delta_{1}, \delta_{2}$ be as in Theorem 1. The proportion of set partitions of $n$ objects, whose block sizes are not in $\mathcal{S}$, is

$$
\frac{B_{n, \mathcal{S}}}{B_{n}}=\exp (-\alpha(r))\left(1+O\left(\frac{\left(\alpha^{\prime}(r)\right)^{2}}{e^{r}}\right)\right)=\exp (-\alpha(r))(1+o(1)),
$$

as $n \rightarrow \infty$, uniformly for sets $\mathcal{S}$ with $\mathcal{S} \cap\left[\delta_{1} r, \delta_{2} r\right]=\emptyset$.

With 1-rough partitions, $\alpha(r)=r$, so that $e^{-r}$ is the main term as well as the relative error term in Corollary 3. This is consistent with Table1, where the relative errors are $<e^{-r}$. Similarly, for 2-rough partitions, the relative errors in Table 2 are $<(1+r)^{2} e^{-r}$, the relative error term in Corollary 3 , 


\begin{tabular}{|c|c|c|c|}
\hline$n$ & $B_{n, 1} / B_{n}$ & $\exp (-r)$ & Rel. Error \\
\hline $2^{2}$ & 0.266667 & 0.300542 & 0.127032 \\
\hline $2^{4}$ & 0.116036 & 0.128325 & 0.105906 \\
\hline $2^{6}$ & 0.045716 & 0.047583 & 0.040834 \\
\hline $2^{8}$ & 0.015896 & 0.016123 & 0.014298 \\
\hline $2^{10}$ & 0.005122 & 0.005146 & 0.004675 \\
\hline $2^{12}$ & 0.001573 & 0.001575 & 0.001456 \\
\hline $2^{14}$ & 0.000468 & 0.000468 & 0.000438 \\
\hline
\end{tabular}

TABLE 1. Proportion of 1-rough set partitions: numerical examples of Corollary 3 for $\mathcal{S}=\{1\}$ and $\alpha(r)=r$, showing the ratio $B_{n, \mathcal{S}} / B_{n}$, the approximation $\exp (-\alpha(r))$ and the relative error $\exp (-\alpha(r)) /\left(B_{n, \mathcal{S}} / B_{n}\right)-1$.

\begin{tabular}{|c|c|c|c|c|}
\hline$n$ & $B_{n, 2} / B_{n}$ & $\exp \left(-r-\frac{r^{2}}{2}\right)$ & Rel. Error & $(1+r)^{2} e^{-r}$ \\
\hline $2^{2}$ & $6.667 \cdot 10^{-2}$ & $1.459 \cdot 10^{-1}$ & 1.1886 & 1.4575 \\
\hline $2^{4}$ & $8.772 \cdot 10^{-3}$ & $1.559 \cdot 10^{-2}$ & 0.7776 & 1.1962 \\
\hline $2^{6}$ & $3.185 \cdot 10^{-4}$ & $4.610 \cdot 10^{-4}$ & 0.4474 & 0.7787 \\
\hline $2^{8}$ & $2.628 \cdot 10^{-6}$ & $3.222 \cdot 10^{-6}$ & 0.2257 & 0.4239 \\
\hline $2^{10}$ & $4.356 \cdot 10^{-9}$ & $4.805 \cdot 10^{-9}$ & 0.1033 & 0.2023 \\
\hline $2^{12}$ & $1.368 \cdot 10^{-12}$ & $1.428 \cdot 10^{-12}$ & 0.0438 & 0.0875 \\
\hline $2^{14}$ & $7.902 \cdot 10^{-17}$ & $8.040 \cdot 10^{-17}$ & 0.0175 & 0.0352 \\
\hline
\end{tabular}

TABLE 2. Proportion of 2-rough set partitions: numerical examples of Corollary 3 for $\mathcal{S}=\{1,2\}$ and $\alpha(r)=r+r^{2} / 2$, showing the ratio $B_{n, \mathcal{S}} / B_{n}$, the approximation $\exp (-\alpha(r))$, the relative error $\exp (-\alpha(r)) /\left(B_{n, \mathcal{S}} / B_{n}\right)-1$, and $\left(\alpha^{\prime}(r)\right)^{2} e^{-r}$, the relative error term in Corollary 3 .

Since $e^{-r}=r / n$ and $r \sim \log n$ by equation (2), the proportion of 1-rough set partitions (i.e. partitions with no singletons) is asymptotic to $(\log n) / n$. Corollary 4 makes that more precise.

Corollary 4. The proportion of 1-rough set partitions of $n$ objects is

$$
\frac{B_{n, 1}}{B_{n}}=\frac{r}{n}\left(1+O\left(\frac{\log n}{n}\right)\right)=\frac{\log (n / \log n)}{n}\left(1+O\left(\frac{\log \log n}{(\log n)^{2}}\right)\right) .
$$

The quantity $B_{n, m}$ appears in [10]. However, [10, Prop. 2] claims that $B_{n, 1} / B_{n} \sim(\log n) /(n e)$, which is false in light of Corollary 4 . Moreover, the asymptotic estimate for $B_{n, m}$ in [10, Prop. 4] is not correct, because $\exp \left(e^{r}\right) \nsim \exp (n / \log n)$ by (2), even though $e^{r}=n / r \sim n / \log n$.

We now turn to an application of Theorem 1. In analogy with practical numbers 8, 11 and practical integer partitions [2, 3, we say that a partition of a set of $n$ objects is practical if its blocks can be combined to form subsets 
of any size between 1 and $n$. Thus, if the partition has $l$ blocks of sizes $a_{1}, a_{2}, \ldots, a_{l}$, the partition is practical if and only if

$$
\left\{\sum_{i=1}^{l} \varepsilon_{i} a_{i}: \varepsilon_{i} \in\{0,1\}\right\}=\{0,1,2,3, \ldots, n\} .
$$

For example, when $n=7$, the set partition $\{\{a\},\{b, c\},\{d, e, f, g\}\}$ is practical, but $\{\{a\},\{b, c, d\},\{e, f, g\}\}$ is not, because the blocks cannot be combined to form a set of size 2 or 5 . Let $P_{n}$ denote the number of practical set partitions and let $I_{n}=B_{n}-P_{n}$, the number of impractical set partitions, of a set of $n$ elements. Define $P_{0}=B_{0}=1$. Partitions which are 1-rough are clearly impractical, since the blocks can not be combined to form a set of size 1 . Theorem 2 shows that, as $n$ grows, almost all impractical set partitions are 1-rough.

Theorem 2. We have

$$
I_{n}=B_{n, 1}\left(1+O\left(\exp \left(-\frac{(\log n)^{2}}{3}\right)\right)\right) .
$$

Combining Theorem 2 with Corollary 4 yields an estimate for $I_{n} / B_{n}$ :

Corollary 5. The proportion of impractical set partitions of $n$ objects is

$$
\frac{I_{n}}{B_{n}}=\frac{r}{n}\left(1+O\left(\frac{\log n}{n}\right)\right)=\frac{\log (n / \log n)}{n}\left(1+O\left(\frac{\log \log n}{(\log n)^{2}}\right)\right) .
$$

\begin{tabular}{|c|c|c|c|}
\hline$n$ & $I_{n} / B_{n}$ & $r / n$ & Relative Error \\
\hline $2^{2}$ & 0.533333 & 0.300542 & -0.436484 \\
\hline $2^{4}$ & 0.141507 & 0.128325 & -0.093156 \\
\hline $2^{6}$ & 0.046743 & 0.047583 & 0.017954 \\
\hline $2^{8}$ & 0.015907 & 0.016123 & 0.013594 \\
\hline $2^{10}$ & 0.005122 & 0.005146 & 0.004670 \\
\hline
\end{tabular}

TABle 3. Proportion of impractical set partitions: numerical examples of Corollary 5, showing the ratio $I_{n} / B_{n}$, the approximation $r / n$ and the relative error $(r / n) /\left(I_{n} / B_{n}\right)-1$.

Note that the relative errors in Table 3 are of a similar size as the main term $r / n$, consistent with the first equation in Corollary 5 ,

Since $P_{n}=B_{n}-I_{n}$, we find that almost all set partitions are practical, as in the case of integer partitions (see [2, 3]).

Corollary 6. The proportion of practical set partitions of $n$ objects is

$$
\frac{P_{n}}{B_{n}}=1-\frac{r}{n}+O\left(\frac{(\log n)^{2}}{n^{2}}\right)=1-\frac{\log (n / \log n)}{n}+O\left(\frac{\log \log n}{n \log n}\right) .
$$




\section{Proof of Theorem 1}

The following lemma gives a recursive formula for the sequence $B_{n, \mathcal{S}}$, which we used to generate the numerical examples in the tables. It is also the basis for deriving the exponential generating function in Lemma 2 ,

Lemma 1. For $n \geq 1$,

$$
B_{n, \mathcal{S}}=\sum_{\substack{0 \leq k \leq n-1 \\
n-k \notin \mathcal{S}}}\left(\begin{array}{c}
n-1 \\
k
\end{array}\right) B_{k, \mathcal{S}}
$$

Proof. We count the number of partitions of the set $\{1,2, \ldots, n\}$, with no block sizes in $\mathcal{S}$. For such a partition, let $k$ be the number of all elements of $\{1,2, \ldots, n\}$ which are not in the block that contains 1 . There are $\left(\begin{array}{c}n-1 \\ k\end{array}\right)$ ways of selecting those elements from $\{2,3, \ldots, n\}$, and for each such selection there are $B_{k, \mathcal{S}}$ set partitions of those elements, with no block size in $\mathcal{S}$. Note that the block containing 1 has $n-k$ elements, so $n-k \notin \mathcal{S}$.

Let

$$
G_{\mathcal{S}}(z)=\sum_{n=0}^{\infty} \frac{B_{n, \mathcal{S}}}{n !} z^{n}
$$

the exponential generating function for the sequence $B_{n, \mathcal{S}}$.

Lemma 2. We have

$$
G_{\mathcal{S}}(z)=\exp \left(e^{z}-1-\alpha(z)\right) .
$$

Proof. It is a standard exercise to derive the differential equation

$$
G_{\mathcal{S}}^{\prime}(z)=G_{\mathcal{S}}(z)\left(e^{z}-\alpha^{\prime}(z)\right)
$$

from the recursive formula in Lemma 1 . Solving that equation for $G_{\mathcal{S}}(z)$ yields the desired result.

Alternatively, the result follows from the general principle in [4, Proposition II.2].

We will need the following upper bound for the $i$-th derivative $\alpha^{(i)}(r)$ :

Lemma 3. Let $\delta_{1}, \delta_{2}$ be as in Theorem 1, and let $I, J \geq 0$ be fixed integers. Uniformly for sets $\mathcal{S}$ with $\mathcal{S} \cap\left[\delta_{1} r, \delta_{2} r\right]=\emptyset$, we have

$$
\alpha^{(i)}(r) \ll_{I, J} \frac{e^{r / 2}}{r^{J}} \quad(0 \leq i \leq I) .
$$

Proof. Write $m=\delta_{1} r$ and $M=\delta_{2} r$. Then

$$
\alpha^{(i)}(r) \leq \sum_{i \leq k \leq m} \frac{r^{k-i}}{(k-i) !}+\sum_{k>M} \frac{r^{k-i}}{(k-i) !}=s_{1}+s_{2},
$$

say. We have

$$
s_{1}=\sum_{0 \leq k \leq m-i} \frac{m^{k}}{k !}\left(\frac{r}{m}\right)^{k} \leq\left(\frac{r}{m}\right)^{m-i} \sum_{0 \leq k \leq m-i} \frac{m^{k}}{k !}<\left(\frac{r}{m}\right)^{m-i} e^{m},
$$


hence

$$
\log s_{1}<m(1-\log (m / r)) .
$$

Since $m / r=\delta_{1}<\eta_{1}$, the definition of $\eta_{1}$ in Theorem 1 implies

$$
(m / r)(1-\log (m / r))<1 / 2-\varepsilon_{1},
$$

for some $\varepsilon_{1}>0$. Combining the last two inequalities shows that

$$
s_{1}<\exp \left(\left(1 / 2-\varepsilon_{1}\right) r\right) \ll_{J} \exp (r / 2) / r^{J} .
$$

Similarly,

$$
s_{2}=\sum_{k>M-i} \frac{M^{k}}{k !}\left(\frac{r}{M}\right)^{k} \leq\left(\frac{r}{M}\right)^{M-i} \sum_{k>M-i} \frac{M^{k}}{k !}<\delta_{2}^{i}\left(\frac{r e}{M}\right)^{M},
$$

hence

$$
\log s_{2}<M(1-\log (M / r))+i \log \delta_{2} .
$$

Since $M / r=\delta_{2}>\eta_{2}$, the definition of $\eta_{2}$ in Theorem 1 implies

$$
(M / r)(1-\log (M / r))<1 / 2-\varepsilon_{2},
$$

for some $\varepsilon_{2}>0$. With $i \leq I$, we get

$$
s_{2} \ll_{I} \exp \left(\left(1 / 2-\varepsilon_{2}\right) r\right) \ll_{I, J} \exp (r / 2) / r^{J} .
$$

Proof of Theorem 1. Let $r$ be given by (11). Cauchy's residue theorem yields

(4) $\frac{B_{n, \mathcal{S}}}{n !}=\frac{1}{2 \pi i} \int_{|z|=r} \frac{G_{\mathcal{S}}(z)}{z^{n+1}} d z=\frac{1}{2 \pi i} \int_{|z|=r} \frac{\exp \left(e^{z}-1-\alpha(z)\right)}{z^{n+1}} d z$.

Writing $z=r e^{i \theta}$, we obtain

$$
\frac{B_{n, \mathcal{S}}}{n !}=\frac{\exp \left(e^{r}-1-\alpha(r)\right)}{2 \pi r^{n}} \int_{-\pi}^{\pi} \exp (h(\theta)) d \theta,
$$

where

$$
h(\theta)=e^{r e^{i \theta}}-e^{r}+\alpha(r)-\alpha\left(r e^{i \theta}\right)-i \theta n .
$$

Our first task is to show that the contribution to the last integral from $\delta \leq|\theta| \leq \pi$ is negligible, where

$$
\delta=\sqrt{12(1+\alpha(r)) e^{-r}} .
$$

We have

$$
|\exp (h(\theta))|=\exp (\operatorname{Re}(h(\theta))) \leq \exp \left(\operatorname{Re} e^{r e^{i \theta}}-e^{r}+2 \alpha(r)\right) .
$$

Since $\operatorname{Re} e^{z}=e^{\operatorname{Re} z} \cos (\operatorname{Im} z)$,

$$
\operatorname{Re} e^{r\left(e^{i \theta}-1\right)}=e^{r(\cos \theta-1)} \cos (r \sin \theta) \leq e^{r(\cos \theta-1)} \leq e^{r(\cos \delta-1)},
$$

for $\delta \leq|\theta| \leq \pi$. Now $\cos \delta \leq 1-\delta^{2} / 3$ for $0 \leq \delta \leq 2$, and $e^{x} \leq 1+x / 2$ for $-1 \leq x \leq 0$. Thus

$$
\operatorname{Re} e^{r\left(e^{i \theta}-1\right)} \leq 1+r(\cos \delta-1) / 2 \leq 1-\frac{r \delta^{2}}{6}
$$


and

$$
\operatorname{Re} e^{r e^{i \theta}}-e^{r}=e^{r}\left(\operatorname{Re} e^{r\left(e^{i \theta}-1\right)}-1\right) \leq-\frac{e^{r} r \delta^{2}}{6}=-2 r(1+\alpha(r)),
$$

for $\delta \leq|\theta| \leq \pi$. Hence

$$
|\exp (h(\theta))| \leq \exp (-2 r(1+\alpha(r))+2 \alpha(r)) \ll \exp (-2 r) .
$$

The contribution to the integral in (5) from $\delta \leq|\theta| \leq \pi$ is thus

$$
\left|\int_{\delta \leq|\theta| \leq \pi} \exp (h(\theta)) d \theta\right| \leq \int_{\delta \leq|\theta| \leq \pi}|\exp (h(\theta))| d \theta \ll e^{-2 r},
$$

which is acceptable.

The second task is to approximate $h(\theta)$ for $|\theta| \leq \delta$ by a Taylor polynomial and show that the error term is negligible. We have

$$
h(\theta)=-i \alpha^{\prime}(r) r \theta-A \frac{\theta^{2}}{2}-i B \frac{\theta^{3}}{6}+O\left(r^{4} e^{r} \theta^{4}\right),
$$

where

$$
A=r^{2} e^{r}\left(1-\alpha^{\prime \prime}(r) e^{-r}\right)+r e^{r}\left(1-\alpha^{\prime}(r) e^{-r}\right),
$$

and

$$
B=e^{r}\left(r^{3}+3 r^{2}+r\right)-\left(r^{3} \alpha^{\prime \prime \prime}(r)+3 r^{2} \alpha^{\prime \prime}(r)+r \alpha^{\prime}(r)\right)=O\left(e^{r} r^{3}\right) .
$$

Since $e^{-i t}=1-i t+O\left(t^{2}\right)$ for all real $t$, we can write $\exp (h(\theta))$ as

$$
e^{-A \theta^{2} / 2}\left(1-i \alpha^{\prime}(r) r \theta-i B \theta^{3} / 6+O\left(\left(r \alpha^{\prime}(r)\right)^{2} \theta^{2}+B^{2} \theta^{6}\right)\right)\left(1+O\left(r^{4} e^{r} \theta^{4}\right)\right),
$$

where the last error term is justified since $r^{4} e^{r} \theta^{4}=O(1)$ for $|\theta| \leq \delta$, by Lemma 3. Multiplying the two factors, and appealing to $r^{4} e^{r} \theta^{4}=O(1)$, shows that $\exp (h(\theta))$ equals

$$
e^{-A \theta^{2} / 2}\left(1-i \alpha^{\prime}(r) r \theta-i B \theta^{3} / 6+O\left(\left(r \alpha^{\prime}(r)\right)^{2} \theta^{2}+B^{2} \theta^{6}+r^{4} e^{r} \theta^{4}\right)\right)
$$

and

$$
\int_{-\delta}^{\delta} \exp (h(\theta)) d \theta=\int_{-\delta}^{\delta} e^{-A \theta^{2} / 2} d \theta+0+0+E
$$

where

$$
E \ll \int_{-\delta}^{\delta} e^{-A \theta^{2} / 2}\left(\left(r \alpha^{\prime}(r)\right)^{2} \theta^{2}+B^{2} \theta^{6}+r^{4} e^{r} \theta^{4}\right) d \theta .
$$

The even central moments of a normal distribution with variance $1 / A$ are given by (see [5, p. 25])

$$
\sqrt{\frac{A}{2 \pi}} \int_{-\infty}^{\infty} e^{-A \theta^{2} / 2} \theta^{2 k} d \theta=\frac{(2 k) !}{(2 A)^{k} k !} \quad(k \geq 0) .
$$

Since $A \gg e^{r} r^{2}$, by Lemma 3 , and $B \ll e^{r} r^{3}$, we obtain

$$
E \sqrt{A} \ll \frac{\left(r \alpha^{\prime}(r)\right)^{2}}{A}+\frac{B^{2}}{A^{3}}+\frac{r^{4} e^{r}}{A^{2}} \ll\left(\alpha^{\prime}(r)\right)^{2} e^{-r}+e^{-r}+e^{-r}
$$


and

$$
\int_{-\delta}^{\delta} \exp (h(\theta)) d \theta=\int_{-\delta}^{\delta} e^{-A \theta^{2} / 2} d \theta+O\left(\frac{1+\left(\alpha^{\prime}(r)\right)^{2}}{\sqrt{A} e^{r}}\right) .
$$

Our third task is to extend the last integral to $(-\infty, \infty)$. We have

$$
\int_{\delta}^{\infty} e^{-A \theta^{2} / 2} d \theta \leq \int_{\delta}^{1} e^{-A \theta^{2} / 2} d \theta+\int_{1}^{\infty} e^{-A \theta / 2} d \theta \leq e^{-A \delta^{2} / 2}+\frac{e^{-A / 2}}{A / 2} \ll e^{-2 r} .
$$

Thus,

$$
\begin{aligned}
\int_{-\delta}^{\delta} \exp (h(\theta)) d \theta & =\int_{-\infty}^{\infty} e^{-A \theta^{2} / 2} d \theta+O\left(\frac{1+\left(\alpha^{\prime}(r)\right)^{2}}{\sqrt{A} e^{r}}\right) \\
& =\sqrt{\frac{2 \pi}{A}}\left(1+O\left(\frac{1+\left(\alpha^{\prime}(r)\right)^{2}}{e^{r}}\right)\right) .
\end{aligned}
$$

To approximate the quantity $A$ by $r(r+1) e^{r}$, we need to estimate $\alpha^{\prime \prime}(r)$ in terms of $\alpha^{\prime}(r)$. We have

$$
\sum_{\substack{1 \leq k-1 \leq 3 r \\ k \in \mathcal{S}}} \frac{r^{k-2}}{(k-2) !} \leq \sum_{\substack{1 \leq k-1 \leq 3 r \\ k \in \mathcal{S}}} \frac{r^{k-2}}{(k-2) !} \cdot \frac{3 r}{k-1} \leq 3 \alpha^{\prime}(r)
$$

and

$$
\sum_{\substack{k-1>3 r \\ k \in \mathcal{S}}} \frac{r^{k-2}}{(k-2) !} \leq \sum_{\substack{k-1>3 r \\ k \in \mathcal{S}}} \frac{(3 r)^{k-2}}{(k-2) !}\left(\frac{1}{3}\right)^{3 r-1}<e^{3 r}\left(\frac{1}{3}\right)^{3 r-1} \leq 3 .
$$

Thus, $\alpha^{\prime \prime}(r) \leq 3+3 \alpha^{\prime}(r)$, and

$$
A=r(r+1) e^{r}\left(1+O\left(\frac{1+\alpha^{\prime}(r)}{e^{r}}\right)\right) .
$$

Theorem 1 now follows from combining the estimates (6), (17) and (18) with equation (5).

\section{Proof of Theorem 2}

Sierpinski [7] and Stewart [9] independently gave the characterization of practical numbers in terms of their prime factors. The following analogue characterizes practical set partitions in terms of their block sizes.

Lemma 4. A set partition with $l$ blocks of sizes $a_{1} \leq a_{2} \leq \ldots \leq a_{l}$ is practical if and only if

$$
a_{i} \leq 1+\sum_{1 \leq j<i} a_{j} \quad(1 \leq i \leq l) .
$$

Proof. Condition (9) is clearly necessary: if $a_{i}>1+\sum_{1 \leq j<i} a_{j}$ for some $1 \leq i \leq l$, then there is no set of size $1+\sum_{1 \leq j<i} a_{j}$ which is the union of different blocks.

To show that (9) is sufficient, we proceed by induction on $l$. The case $l=1$ is obvious. Assume that (9) implies that the corresponding set partition with 
block sizes $a_{1} \leq \ldots \leq a_{l}$ is practical for some $l \geq 1$. Assume a set partition with block sizes $a_{1} \leq \ldots \leq a_{l} \leq a_{l+1}$ satisfies (9), with $l$ replaced by $l+1$. The set of sizes of subsets obtained from combining different blocks is

$$
A:=\left\{\sum_{i=1}^{l+1} \varepsilon_{i} a_{i}: \varepsilon_{i} \in\{0,1\}\right\}=\left\{\sum_{i=1}^{l} \varepsilon_{i} a_{i}: \varepsilon_{i} \in\{0,1\}\right\}+\left\{0, a_{l+1}\right\}
$$

By the inductive hypothesis, $A=\left\{1,2,3, \ldots, \sum_{i=1}^{l} a_{i}\right\}+\left\{0, a_{l+1}\right\}$, and since $a_{l+1} \leq 1+\sum_{1 \leq j<l+1} a_{j}$, we have $A=\left\{1,2,3, \ldots, \sum_{i=1}^{l+1} a_{i}\right\}$.

The following functional equation is the analogue of [11, Lemma 2.3] for practical numbers and of [2, Lemma 5] for practical integer partitions. Other analogues include polynomials over finite fields [12, Lemma 5] and permutations [12, Lemma 11].

Lemma 5. For $n \geq 0$,

$$
B_{n}=\sum_{k=0}^{n}\left(\begin{array}{l}
n \\
k
\end{array}\right) P_{k} B_{n-k, k+1} .
$$

Proof. Given any partition of $n$ objects with block sizes $a_{1} \leq a_{2} \leq \ldots \leq a_{l}$, let $l_{0}$ be the largest index such that

$$
a_{i} \leq 1+\sum_{1 \leq j<i} a_{j} \quad\left(1 \leq i \leq l_{0}\right)
$$

and let $l_{0}=0$ if $a_{1}>1$. By Lemma 4 , the blocks of sizes $a_{1} \leq \ldots \leq a_{l_{0}}$ form a practical set partition of a set with $k:=\sum_{1 \leq j \leq l_{0}} a_{j}$ elements. Since $l_{0}$ was maximal, the remaining blocks have sizes $k+1<a_{l_{0}+1} \leq \ldots \leq a_{l}$ and form a $(k+1)$-rough set partition of a set with $n-k$ elements. The lemma now follows since $0 \leq k \leq n$ and there are $\left(\begin{array}{l}n \\ k\end{array}\right)$ ways to choose the $k$ elements that belong to the practical set partition.

Lemma 6. For $n \geq 1$,

$$
I_{n}=B_{n, 1}+\sum_{k=1}^{\left\lfloor\frac{n-2}{2}\right\rfloor}\left(\begin{array}{l}
n \\
k
\end{array}\right) P_{k} B_{n-k, k+1} .
$$

Proof. In Lemma 5, the term corresponding to $k=0$ is $B_{n, 1}$, since $P_{0}=1$. The term corresponding to $k=n$ is $P_{n}$, since $B_{0, n+1}=1$. If $(n-2) / 2<$ $k<n$, then $0<n-k<k+2$, so $B_{n-k, k+1}=0$. The result now follows since $I_{n}=B_{n}-P_{n}$.

For the remainder of this section, we write

$$
\beta_{m}(z)=\sum_{j=0}^{m} \frac{z^{j}}{j !}
$$


Lemma 7. For $n \geq 1, m \geq 0$, we have

$$
B_{n, m} \leq \frac{n ! \exp \left(e^{r}-\beta_{m}(r)\right)}{r^{n}}
$$

Proof. With $\mathcal{S}=\{1,2,3, \ldots, m\}$, the integrand in equation (44) satisfies

$$
\left|\frac{\exp \left(e^{z}-\beta_{m}(z)\right)}{z^{n+1}}\right|=\frac{\exp \left(\operatorname{Re} \sum_{k=m+1}^{\infty} \frac{z^{k}}{k !}\right)}{|z|^{n+1}} \leq \frac{\exp \left(\sum_{k=m+1}^{\infty} \frac{r^{k}}{k !}\right)}{r^{n+1}},
$$

and therefore

$$
\frac{B_{n, m}}{n !} \leq \frac{1}{2 \pi} 2 \pi r \frac{\exp \left(\sum_{k=m+1}^{\infty} \frac{r^{k}}{k !}\right)}{r^{n+1}}=\frac{\exp \left(e^{r}-\beta_{m}(r)\right)}{r^{n}} .
$$

Proof of Theorem 2. We write $b_{n, k}=B_{n, k} / n !, p_{n}=P_{n} / n$ ! and $i_{n}=I_{n} / n$ !. Lemma 6 says that

$$
0 \leq i_{n}-b_{n, 1}=\sum_{k=1}^{N} p_{k} b_{n-k, k+1} \leq \sum_{k=1}^{N} b_{k} b_{n-k, k+1},
$$

where $N=\left\lfloor\frac{n-2}{2}\right\rfloor$. To establish Theorem 2, we need to show that the last sum satisfies

$$
\sum_{k=1}^{N} b_{k} b_{n-k, k+1} \ll b_{n, 1} \exp \left(-(\log n)^{2} / 3\right) .
$$

We write

$$
\sum_{k=1}^{N} b_{k} b_{n-k, k+1}=\sum_{1 \leq k \leq L}+\sum_{L<k \leq M}+\sum_{M<k \leq N}=S_{1}+S_{2}+S_{3},
$$

say, where $L=(\log n)^{3 / 2}$ and $M=\left\lfloor\frac{n-4}{3}\right\rfloor$. Let

$$
g(n)=n \log (r(n))-\frac{n}{r(n)} \sim n \log \log n,
$$

by (2). It is easy to verify that

$$
g^{\prime}(n)=\log r(n) \sim \log \log n .
$$

Theorem 1 shows that

$$
b_{n, 1}=\exp \left(e^{r}-n \log r+O(r)\right)=\exp (-g(n)+O(\log n)),
$$

since $e^{r}=n / r$. Similarly, Lemma 7 shows that, for $n \geq 1$ and $m \geq 0$,

$$
b_{n, m} \leq \exp \left(-g(n)-\beta_{m}(r(n))\right) .
$$

It follows that

$$
\log \left(b_{k} b_{n-k, k+1}\right) \leq-g(k)-g(n-k)-\beta_{k+1}(r(n-k)) .
$$


For $S_{1}$, we have $1 \leq k \leq L=(\log n)^{3 / 2}$, so (12) and (11) yield

$$
\begin{aligned}
\log \left(b_{k} b_{n-k, k+1}\right) & \leq 0-g(n-L)-\beta_{2}(r(n / 2)) \\
& \leq-g(n)+O(L \log \log n)-(\log n)^{2} /(2+\varepsilon) \\
& \leq-g(n)-(\log n)^{2} /(2+2 \varepsilon),
\end{aligned}
$$

for any $\varepsilon>0$ and $n \geq n_{0}(\varepsilon)$. Hence

$$
\begin{aligned}
S_{1} & \leq L \exp \left(-g(n)-(\log n)^{2} /(2+2 \varepsilon)\right) \\
& \leq b_{n, 1} \exp \left(-(\log n)^{2} /(2+3 \varepsilon)\right) .
\end{aligned}
$$

When $L<k \leq N$, we have

$$
\begin{aligned}
0 \leq e^{r(n-k)}-\beta_{k+1}(r(n-k)) & =\sum_{j=k+2}^{\infty} \frac{(r(n-k))^{j}}{j !} \leq \sum_{j=k+2}^{\infty} \frac{k^{j}}{j !}\left(\frac{r(n)}{k}\right)^{j} \\
& \leq\left(\frac{r(n)}{L}\right)^{k+2} e^{k}=o(1),
\end{aligned}
$$

as $n \rightarrow \infty$. Since $e^{r(n-k)}=(n-k) / r(n-k)$, (12) yields

$$
\begin{aligned}
\log \left(b_{k} b_{n-k, k+1}\right) & \leq-g(k)-g(n-k)-\frac{n-k}{r(n-k)}+o(1) \\
& =-f(n, k)+o(1)
\end{aligned}
$$

say. We claim that $f(n, k)$ is decreasing (and hence $-f(n, k)$ is increasing) in $k$, for $1 \leq k \leq n / 2$. Indeed, since $r(n)$ is increasing, (11) shows that $g(k)+g(n-k)$ is decreasing in $k$, for $1 \leq k \leq n / 2$. Moreover, $e^{r(n-k)}$ is clearly decreasing in $k$. Thus,

$$
\begin{aligned}
S_{2} & \leq M \exp (-f(n, M)+o(1)) \\
& \leq b_{n, 1} \exp (g(n)-f(n, n / 3)+O(\log n)) \\
& \leq b_{n, 1} \exp (-0.03 n / \log n),
\end{aligned}
$$

for $n$ sufficiently large. The last inequality, whose derivation is not difficult but somewhat tedious, follows from (2).

Finally, when $M<k \leq N$, we have $n-k \geq k+2>(n-k) / 2$. Thus, $B_{n-k, k+1}=1$ and $b_{n-k, k+1}=1 /(n-k)$ !. Stirling's approximation, in the form $\log (n !)=n(\log n-1)+O(\log n)$, yields

$$
\begin{aligned}
\log \left(b_{k} b_{n-k, k+1}\right) & \leq-g(k)-\log ((n-k) !) \\
& \leq 0-(n-k)(\log (n-k)-1)+O(\log n) \\
& \leq-(n / 2)(\log (n / 2)-1)+O(\log n) .
\end{aligned}
$$

Hence

$$
\begin{aligned}
S_{3} & \leq N \exp (-(n / 2)(\log (n / 2)-1)+O(\log n)) \\
& \leq b_{n, 1} \exp (g(n)-(n / 2)(\log (n / 2)-1)+O(\log n)) \\
& \leq b_{n, 1} \exp \left(-\frac{n \log n}{3}\right)
\end{aligned}
$$


for $n$ sufficiently large.

The estimates (13), (14) and (15) show that (10) holds, which completes the proof of Theorem 2 .

\section{REFERENCES}

[1] R. M. Corless, G. H. Gonnet, D. E. G. Hare, D. J. Jeffrey, D. E. Knuth, On the Lambert $W$ function, Adv. Comput. Math. 5 (1996), no. 4, 329-359.

[2] J. Dixmier and J.-L. Nicolas, Partitions without small parts. Number theory, Vol. I (Budapest, 1987), 9-33, Colloq. Math. Soc. János Bolyai, 51, North-Holland, Amsterdam, 1990.

[3] P. Erdős and M. Szalay, On some problems of J. Dénes and P. Turán, Studies in pure mathematics, 187-212, Birkhäuser, Basel, 1983.

[4] P. Flajolet and R. Sedgewick, Analytic Combinatorics, Cambridge Univ. Press, 2009.

[5] J. Patel and C. Read, Handbook of the Normal Distribution, Second Edition, CRC Press, 1996.

[6] L. Moser and M. Wyman, An asymptotic formula for the Bell numbers, Transactions of the Royal Society of Canada 49 (1955) 49-54.

[7] W. Sierpinski, Sur une propriété des nombres naturels, Ann. Mat. Pura Appl. (4) 39 (1955), 69-74.

[8] A. K. Srinivasan, Practical numbers, Current Sci. 17 (1948), 179-180.

[9] B. M. Stewart, Sums of distinct divisors, Amer. J. Math. 76 (1954), 779-785.

[10] C. Wang and I. Mezö, Some limit theorems with respect to constrained permutations and partitions, Monatsh. Math. 182 (2017), no. 1, 155-164.

[11] A. Weingartner, Practical numbers and the distribution of divisors, Q. J. Math. 66 (2015), 743-758.

[12] A. Weingartner, On the degrees of polynomial divisors over finite fields, Math. Proc. Cambridge Philos. Soc. 161 (2016), no. 3, 469-487.

Department of Mathematics, Southern Utah University, Cedar City, Utah 84720

E-mail address: joshua.e.culver@gmail.com

Department of Mathematics, Southern Utah University, Cedar City, Utah 84720

E-mail address: weingartner@suu.edu 